\title{
Evaluating Caregiver Burden as a Predictive Factor for Patient Outcomes in Patients Receiving Anti- cancer Systemic Therapy at a Tertiary Institution in Cebu City
}

Clarence Aaron Cheng Sy ( $\sim$ clarence.sy@obf.ateneo.edu )

Chong Hua Hospital https://orcid.org/0000-0002-3617-4518

\section{Research Article}

Keywords: Caregiver burden, Cancer patients, Chemotherapy, Quality of life

Posted Date: November 30th, 2021

DOI: https://doi.org/10.21203/rs.3.rs-1025935/v1

License: (9) This work is licensed under a Creative Commons Attribution 4.0 International License.

Read Full License 


\section{Abstract}

Background: Though found to be a common occurrence in individuals caring for patients with debilitating illnesses such as cancer, caregiver burden remains underprioritized, and its relationship with patient outcomes is only sparingly described.

Methodology: This cross-sectional, observational study included 50 patient-caregiver pairs receiving anticancer systemic therapy at a tertiary hospital in Cebu City. Sociodemographic and clinical characteristics were obtained. Patient quality of life was measured using the EQ-5D-5L Questionnaire, while caregiver burden was assessed with the Zarit Burden Interview. The association between caregiver burden and patient outcomes were examined using multivariate logistic regression models and bivariate analysis.

Results: Patients included in the study were predominantly females, middle-aged to elderly, and in advanced stages of disease, whereas caregivers were mostly female, and younger in relation. The usual patient-caregiver relationships were spousal and parental. Educational attainment, employment status and income varied among caregivers, and were significantly associated with caregiver burden, as were patient age, a lung primary, and limitations in patient mobility, self-care and ability to perform usual activities. On the other hand, higher levels of caregiver burden were linked to quality of life impairment, increased anxiety or depression, and more frequent hospital admissions for patients.

Discussion: The level of care in patients requiring continuous assistance often leads to an imbalance of care demands relative to the caregiver's personal time and space, roles and resources, the latter being particularly relevant in the third-world setting where healthcare expenditure remains largely out-of-pocket. Patients likewise suffer from the burden they unwillingly impose on caregivers, with self-perceived burden contributing significantly to patient anxiety and depression, and influencing therapeutic choices in the direction of palliative care over curative therapy. Additionally, a higher level of caregiver burden was found to compromise the quality of care, and increase reliance on health systems, often leading to more frequent hospital admissions, perpetuating a vicious cycle of demand and burden.

Conclusions: Financial standing, which can be influenced by educational attainment, employment status and income, was found to be significantly associated with caregiver burden. Patient-related factors such as age, a lung primary, and assistance requirement were also associated factors. Conversely, caregiver burden was found to influence patient quality of life via the impairment of mobility, self-care and ability to perform activities of daily living, and an increase in anxiety or depression. Higher levels of caregiver burden were also found to be associated with a higher frequency of hospital admissions for the patient.

\section{Introduction}

Providing care for patients with debilitating illness such as cancer often falls on family members who lack proper training for care. The chronic and progressive nature of the disease requires adapting to the different levels of care required with time. This is often perceived as a chronic stressor, which brings about ill effects on the caregiver. ${ }^{1}$ 
Caregiver burden (CB) is defined as a "multidimensional biophysical reaction resulting from an imbalance of care demands relative to the caregivers' personal time, social roles, physical and emotional states, financial resources, and formal care resources given by the other roles they have fulfill." ${ }^{2}$

Studies have depicted an inverse relationship between increased levels of patient care with caregiver outcomes, which include quality of life, health-seeking behaviors, and effective partnership. ${ }^{1}$ CB has also been found to significantly affect medical decisions regarding treatment. ${ }^{3}$ However, research detailing the relationship between this phenomenon and how it translates to patient outcomes has been limited. Available studies have shown that increased levels of CB were found to be related to increased patient anxiety and depression, as well as increased use of healthcare facilities. ${ }^{2}$

Identifying caregivers with suboptimal well-being may thus help to pinpoint care recipients with poor outcomes and impending increases in healthcare costs. Screening for well-being in caregivers therefore does not only benefit them, but also helps to target interventions to reduce healthcare use by the care recipient. ${ }^{4}$

\section{OBJECTIVES}

\section{General Objective}

To evaluate the relationship between caregiver burden and patient outcomes among caregivers of patients with cancer receiving anti-cancer systemic therapy

\section{Specific Objectives}

1. To identify demographic characteristics of patients receiving anti-cancer systemic therapy at the institution under study, along with those of their caregivers

2. To define the association between caregiver characteristics and their levels of burden

3. To determine the association between patient characteristics and quality of life with the caregiver's level of burden

4. To demonstrate the association between caregiver burden and patient outcomes

\section{Methodology}

\section{Study Design}

Single-center cross-sectional study

\section{Study Setting}

Private, tertiary-level hospital in Cebu City, from March to November, 2021

\section{Study Population}


This study included pairs of patients and caregivers, $n=50$. Patients included were diagnosed with cancer, aged 18 years and above, who received anti-cancer systemic therapy at the hospital's HematologyOncology unit. Caregivers were family members, aged 18 years and above. Pre-requisites for both groups included adequate physical condition and mental cognition to complete a questionnaire.

\section{Data Collection}

Following an in-depth discussion on the purpose of the study, and the nature of the pre-validated tools to be used, consent to participate in the study was sought. Once consent was given, respective questionnaires were handed individually to patients and to caregivers, each to be answered separately from one another. Instructions on how to answer the questionnaire were provided verbally and in writing. The researcher was present during the accomplishment of questionnaires to address any questions and concerns the participants may have had.

Patient quality of life was assessed using the EQ-5D-5L questionnaire, which provides a descriptive system comprising of five dimensions: mobility, self-care, activities of daily living (ADL), pain/discomfort, and anxiety/depression. Each patient was asked to indicate his or her self-perceived state of health by ticking the box beside the appropriate statement in each of the five dimensions. Caregiver burden, on the other hand, was measured with the Zarit Burden Interview, which is a 22-item questionnaire, with a 5-point grading scale. The Zarit burden index score is derived from the summation of scores for all items.

\section{Statistical Analyses}

Descriptive statistics were used to demonstrate patient characteristics and their quality of life; the same tool was also used to describe caregiver characteristics and levels of burden. To determine the association between patient-related factors and the level of caregiver burden, ordinal logistic regression analysis was performed. Following that, in order to define the association between the level of caregiver burden and patient outcomes, a bivariate analysis was performed using the Pearson $r$ product moment correlation. Across the statistical analyses performed, a p-value of less than 0.05 was regarded significant.

\section{Results}

A total of 50 participant pairs of patients and caregivers were included in the study. The demographic characteristics of participants is presented separetely in Supplementary Table 1. Of the patients who participated, the average age was $51.86 \pm 13.39$ years, of which $62 \%$ were female and $38 \%$ were male. The majority of patients were married or living with partner (56\%). As for educational attainment, the majority were college graduates, though employment status varied across the board, as did estimated monthly income. Regarding the primary disease sites, patients were diagnosed with varying malignancies, of which breast and gastrointestinal sites comprised the majority. As can be expected from their need for anti-cancer systemic therapy, most patients were in Stages III and IV disease (together comprising $76 \%$ ). The most common patient-caregiver relationships were spousal (32\%) and parental (30\%). Of the participant caregivers, the mean age was found to be younger at $41.3 \pm 14.36$, of which $70 \%$ 
were female. Educational attainment, employment status and estimated monthly income varied across caregivers, with most earning college degrees, most of them employed, and the majority earning upwards of 50,000 PHP per month.

As regards the quality of life per the EQ-5D-5L tool, most patients in the study showed slight limitation of mobility, no problems with self-care, moderate limitations in performing ADLs independently, and showed slight pain or discomfort, as well as slight anxiety or depression. Regarding CB, the level of burden in study participants was found to range from moderate to severe, with relatively equal distribution among the three levels. None of the participants reported low levels of burden. The data for patients and caregivers are presented separately in Supplementary Tables 2 and 3, respectively.

Of the caregiver characteristics, results demonstrated that educational attainment, employment status and estimated monthly income were the factors significantly associated with the level of $\mathrm{CB}$, while age and sex were not significantly associated with it (Table 1).

Table 1. The Association between caregiver demographic characteristics and level of burden, $n=50$ 


\begin{tabular}{|c|c|c|c|c|}
\hline \multicolumn{5}{|l|}{ Response Information } \\
\hline Variable & \multicolumn{3}{|l|}{ Value } & Count \\
\hline \multirow[t]{5}{*}{ Level of Caregiver Burden } & \multicolumn{3}{|l|}{ Severe } & 15 \\
\hline & \multicolumn{3}{|l|}{ High } & 18 \\
\hline & \multicolumn{3}{|l|}{ Moderate } & 17 \\
\hline & \multicolumn{3}{|l|}{ Low } & 0 \\
\hline & \multicolumn{3}{|l|}{ Total } & 50 \\
\hline \multicolumn{5}{|l|}{ Logistic Regression Table } \\
\hline Caregiver Factor & Coefficient & Odds Ratio & Z Value & P-Value \\
\hline Age & 0.00694 & 1.01 & 0.38 & 0.705 \\
\hline \multicolumn{5}{|c|}{ Sex (Reference Level: Female) } \\
\hline Male & 0.1361 & 1.15 & 0.24 & 0.811 \\
\hline \multicolumn{5}{|c|}{ Education (Reference Level: College Graduate) } \\
\hline High school Graduate & 1.8371 & 6.28 & 3.13 & 0.002 * \\
\hline \multicolumn{5}{|c|}{ Employment Status (Reference Level: Full or Part-time) } \\
\hline Retired & 0.6171 & 1.85 & 0.73 & 0.462 \\
\hline Not employed & 1.3255 & 3.76 & 2.23 & 0.026 * \\
\hline \multicolumn{5}{|c|}{ Estimated Monthly Income of Family (Reference Level: $<\mathbf{5 0 , 0 0 0 ~ P H P ) ~}$} \\
\hline$>100,000$ PHP & -1.9980 & 0.14 & -2.57 & 0.010 * \\
\hline $50,000-100,000$ PHP & -3.1659 & 0.04 & -3.68 & $<0.001$ * \\
\hline
\end{tabular}

The coefficients and odds ratios of the significant factors suggest that the odds of high or severe burden is:

- Approximately 6 times higher in those whose highest education attainment was a high school graduation versus those who completed a college degree

- Approximately 4 times higher in caregivers who were unemployed than those who were working fullor part-time

- Lower with caregivers with estimated monthly incomes greater than 100,000 PHP than those making less than 50,000 PHP per month 
Of patient factors influencing caregiver burden (Table 2), results indicated that older patient age and lung cancer as the primary site of disease were associated with higher level of CB. Time since diagnosis, which corresponds to the duration of care, was not found to influence CB.

Table 2. The Association between Patient-Related Factors and Level of Caregiver Burden, $n=50$

\begin{tabular}{|c|c|c|c|c|}
\hline \multicolumn{5}{|l|}{ Response Information } \\
\hline Variable & \multicolumn{3}{|l|}{ Value } & Count \\
\hline \multirow[t]{5}{*}{ Level of Caregiver Burden } & \multicolumn{3}{|l|}{ Severe } & 15 \\
\hline & \multicolumn{3}{|l|}{ High } & 18 \\
\hline & \multicolumn{3}{|l|}{ Moderate } & 17 \\
\hline & \multicolumn{3}{|l|}{ Low } & 0 \\
\hline & \multicolumn{3}{|l|}{ Total } & 50 \\
\hline \multicolumn{5}{|l|}{ Logistic Regression Table } \\
\hline Patient Factors & Coefficient & Odds Ratio & Z Value & $P$-Value \\
\hline \multicolumn{5}{|c|}{ Primary Site (Reference Level: Breast) } \\
\hline Gastrointestinal & 1.0663 & 2.90 & 1.45 & 0.146 \\
\hline Genitourinary & -0.2325 & 0.79 & -0.25 & 0.806 \\
\hline Hematologic & 0.8869 & 2.43 & 1.10 & 0.271 \\
\hline Lung & 3.203 & 24.60 & 2.52 & $0.012^{*}$ \\
\hline Other & 0.887 & 2.43 & 0.75 & 0.455 \\
\hline Age & 0.05899 & 1.06 & 2.67 & $0.008^{*}$ \\
\hline \multicolumn{5}{|l|}{ Sex (Reference Level: Female) } \\
\hline Male & 0.9512 & 2.59 & 1.72 & 0.085 \\
\hline \multicolumn{5}{|c|}{ Duration of Illness (Reference Level: < 6 months since diagnosis) } \\
\hline 6-12 months since diagnosis & 0.2088 & 1.23 & 0.37 & 0.714 \\
\hline$>12$ months since diagnosis & 0.2088 & 1.23 & 0.27 & 0.786 \\
\hline
\end{tabular}

The coefficients and odds ratios of the significant factors suggest that the odds of a caregiver experiencing high or severe burden is:

- Approximately 25 times higher when caring for lung cancer patients than in breast cancer patients 
- Higher when caring for elderly patients than for younger cancer patients

When accounting for patient quality of life and number of admissions, a significant association was found between certain components comprising a patient's quality of life (issues with mobility, self-care and performance of ADLs) and CB. These are presented below in Table 3.

Table 3. The association between patient quality of life and number of admissions with the level of caregiver burden, $n=50$ 


\begin{tabular}{|c|c|c|c|c|}
\hline \multicolumn{5}{|l|}{ Response Information } \\
\hline Variable & \multicolumn{3}{|l|}{ Value } & Count \\
\hline \multirow[t]{5}{*}{ Level of Caregiver Burden } & \multicolumn{3}{|l|}{ Severe } & 15 \\
\hline & \multicolumn{3}{|l|}{ High } & 18 \\
\hline & \multicolumn{3}{|l|}{ Moderate } & 17 \\
\hline & \multicolumn{3}{|l|}{ Low } & 0 \\
\hline & \multicolumn{3}{|l|}{ Total } & 50 \\
\hline \multicolumn{5}{|l|}{ Logistic Regression Table } \\
\hline Patient Factor & Coefficient & Odds Ratio & Z Value & P-Value \\
\hline Number of admissions since diagnosis & 0.2531 & 1.29 & 1.39 & 0.165 \\
\hline \multicolumn{5}{|l|}{ Patients' Quality of Life (per EQ-5D-5L) } \\
\hline \multicolumn{5}{|l|}{ Mobility (Reference Level: No Problems) } \\
\hline Slightly Limited & 2.6154 & 13.67 & 3.39 & $0.001^{*}$ \\
\hline Moderately Limited & 3.3134 & 27.48 & 3.72 & $0.000^{*}$ \\
\hline Severely Limited & 1.657 & 5.24 & 1.38 & 0.167 \\
\hline Unable & -20 & 0.00 & -0.00 & 0.999 \\
\hline \multicolumn{5}{|c|}{ Capability of Self-Care (Reference Level: No Problems) } \\
\hline Slightly Limited & 1.7047 & 5.50 & 2.60 & $0.009 *$ \\
\hline Moderately Limited & 22 & $4.78 \mathrm{E}+09$ & 0.00 & 0.999 \\
\hline Severely Limited & - & - & -- & - \\
\hline Unable & -21 & 0.00 & -0.00 & 0.999 \\
\hline \multicolumn{5}{|c|}{ Ability to Perform ADLs (Reference Level: No Problems) } \\
\hline Slightly Limited & 1.726 & 5.62 & 1.45 & 0.148 \\
\hline Moderately Limited & 3.723 & 41.37 & 2.92 & $0.003^{*}$ \\
\hline Severely Limited & 5.219 & 184.70 & 3.32 & $0.001^{*}$ \\
\hline Unable & 3.153 & 23.40 & 2.29 & $0.022^{*}$ \\
\hline \multicolumn{5}{|c|}{ Pain or Discomfort (Reference Level: No Pain or Discomfort) } \\
\hline Slight Pain or Discomfort & -0.233 & 0.79 & -0.17 & 0.862 \\
\hline
\end{tabular}




\begin{tabular}{|lllll|}
\hline \multicolumn{5}{|l}{ Response Information } \\
\hline Moderate Pain or Discomfort & 0.188 & 1.21 & 0.13 & 0.894 \\
\hline Severe Pain or Discomfort & 0.636 & 1.89 & 0.40 & 0.692 \\
\hline Extreme Pain or Discomfort & -22 & 0.00 & -0.00 & 0.999 \\
\hline Anxiety or Depression (Reference Level: Neither Anxious nor Depressed) & \\
\hline Slightly Anxious or Depressed & 0.1053 & 1.11 & 0.12 & 0.907 \\
\hline Moderately Anxious or Depressed & 1.2220 & 3.39 & 1.39 & 0.163 \\
\hline Severely Anxious or Depressed & 0.813 & 2.26 & 0.54 & 0.592 \\
\hline Extremely Anxious or Depressed & 1.896 & 6.66 & 1.19 & 0.234 \\
\hline Note: Significant at 0.05 using ordinal logistic regression analysis & & \\
\hline
\end{tabular}

The coefficients and odds ratios of the significant factors suggest that the odds that the caregiver will experience severe or high burden is:

- Approximately 14 times higher in caregivers of patients with slightly limited mobility than those caregivers of patients with no problems in mobility, and approximately 27 times higher in those of patients with moderately limited mobility

- Approximately 6 times higher in caregivers of cancer patients with slightly limited self-care than those of cancer patients with no problems in self-care

- Approximately 41 times higher in caregivers of patients with moderate limitation of independent $A D L s$ than those of patients with no problems in capability to perform ADLs independently, approximately 185 times higher in those of patients with severely limited ability to perform independent $A D L s$; and approximately 23 times higher in those of patients unable to perform $A D L s$ independently

- Approximately 23 times higher with those caregivers of cancer patients who are unable to perform $A D L s$ than those caregivers of cancer patients with no problems in capability to perform $A D L s$

A bivariate analysis was performed to demonstrate the inverse of this relationship. The results are summarized below in Tables 4 and 5 .

Table 4. The Bivariate Analysis of the Level of Caregiver Burden and Patient Outcomes, $n=50$ 


\begin{tabular}{|ll|}
\hline Patient Outcomes & Caregiver Burden (ZBI Scores) \\
\hline Number of Admissions since Diagnosis & $0.168(0.245)$ \\
\hline Quality of Life & \\
\hline Mobility & $0.319(0.024)^{\star}$ \\
\hline Capability of Self-Care & $0.147(0.308)$ \\
\hline Ability to Perform ADLs & $0.462(0.001)^{\star}$ \\
\hline Pain or Discomfort & $0.071(0.626)$ \\
\hline Anxiety or Depression & $0.289(0.042)^{\star}$ \\
\hline Note: Significant at 0.05 using Pearson r product moment correlation \\
\hline
\end{tabular}

The results demonstrate that higher levels of $\mathrm{CB}$ were associated with the impairment of patient mobility, and ability to perform independent ADLs, as well as increased anxiety and depression.

Table 5. The Association between the Patients' Number of Admissions since Diagnosis and the Level of Caregiver Burden, $n=50$ 


\begin{tabular}{|c|c|c|c|c|}
\hline \multicolumn{5}{|l|}{ Response Information } \\
\hline Variable & \multicolumn{3}{|l|}{ Value } & Count \\
\hline \multirow[t]{10}{*}{ Number of Admissions since Diagnosis } & \multicolumn{3}{|l|}{0} & 1 \\
\hline & \multicolumn{3}{|l|}{1} & 11 \\
\hline & \multicolumn{3}{|l|}{2} & 18 \\
\hline & \multicolumn{3}{|l|}{3} & 11 \\
\hline & \multicolumn{3}{|l|}{4} & 5 \\
\hline & \multicolumn{3}{|l|}{5} & 2 \\
\hline & \multicolumn{3}{|l|}{6} & 0 \\
\hline & \multicolumn{3}{|l|}{7} & 1 \\
\hline & \multicolumn{3}{|l|}{8} & 1 \\
\hline & \multicolumn{3}{|l|}{ Total } & 50 \\
\hline \multicolumn{5}{|l|}{ Logistic Regression Table } \\
\hline Patient Factor & Coefficient & Odds Ratio & Z Value & P-Value \\
\hline \multicolumn{5}{|c|}{ Level of Caregiver Burden (Reference Level: Severe) } \\
\hline High & 0.8705 & 2.39 & 1.36 & 0.173 \\
\hline Moderate & 1.3335 & 3.79 & 2.01 & $0.044^{*}$ \\
\hline Low & -- & - & - & - \\
\hline
\end{tabular}

When looking into the relationship between CB and hospital admissions for the patient, it was likewise found that the odds of patient admission increases approximately 4 times in caregivers who experience even moderate levels of burden.

\section{Discussion}

Caregivers who participated in the study were found to be generally younger than their patient counterparts, were mostly female, and in spousal or parent-child relationships with their patients. The predominating demographic characteristics are believed to be reflective of the cultural milieu of Asian communities, with the responsibility of care often falling into the hands of younger, usually female, family members. These individuals face the dilemma of being thrown into a labor-intensive role without receiving any formal training in care provision, leaving them feeling unprepared, in addition to the 
challenge of witnessing a loved one struggling with serious illness, putting them at risk for depression and anxiety, and attenuating their ability to provide care. ${ }^{5}$

Among caregiver characteristics, the study identified educational attainment, status of employment, and income as significant contributors to CB. This mirrors some of the findings in the systematic review conducted by Ge and Mordiffi, where it was found that younger caregivers were more likely to experience psychological distress as a result of less secure financial status, and activity restriction. ${ }^{7}$ This concern is heightened in the setting of a health system that remains predominantly reliant on out-of-pocket expenses rather than state-subsidized care.

In a study by Weitzner et al, in addition to caregiver characteristics, illness-related indices (such as disease site, stage, duration and prognosis) were also found to play a significant role. ${ }^{6}$ This was consistent with the findings from our study with lung cancer being the primary site most associated with CB. Patient age was likewise a contributory factor. More advanced cancer often translates to a higher complexity of treatment regimens, increased patient disability from both disease and treatment, and poorer prognosis; and this effect is magnified in older age, culminating in a higher demand for caregiving, eventually leading to caregiver burden. Unlike the Weitzner study, however, duration of illness was not found to be significantly associated with CB in our study, which might be attributed to either the relatively smaller sample size or the presumed adoption of coping mechanisms to deal with feelings of burden over time.

Additionally, literature cites the patient's requirement for assistance as a primary factor contributing to $\mathrm{CB}$, given that this involves an increased amount of time and a higher level of engagement. In our study, we found this to be true as well, with impairments in patient mobility and self-care, and the requirement of assistance in performing ADLs contributing significantly. ${ }^{7}$ However, our study did not demonstrate a strong association between patient anxiety or depression with CB.

On the other hand, when examining the inverse relationship via bivariate analysis, the study found that higher levels of CB were linked to the impairment of patient mobility, and a decreased ability to perform $A D L s$ independently. Apart from its ill effects on the caregiver (including decreased quality of life, heathseeking behaviors, effective partnership), CB is of particular concern from a clinical standpoint because caregivers who are "burned out" are at risk of being unable to continue providing care for the patient, ${ }^{1}$ which, in turn, will have ill effects on the patient's condition, perpetuating a vicious cycle of patient deterioration and worsening caregiver burden. Studies also suggest that caregiver burden affects medical decisions, including the choice of placement for end-of-life care or patient institutionalization. In a crosssectional study by Ji et al, caregivers who scored higher on questionnaires probing for burden were more likely to prefer palliative care over life-sustaining treatment, meaning that the likelihood of preferring palliation increased as the burden score increased. ${ }^{3}$

Our study likewise demonstrated a strong association between higher levels of caregiver burden and increased patient anxiety or depression. As debilitating illnesses such as cancer constrain a patient's 
ability for self-care, the responsibility of care shifts to others, which the patient hands over with reluctance. Self-perceived burden (SPB) is a multi-dimensional construct arising from a patient's feelings of dependence, and the resulting frustration and worry, which then lead to negative feelings of guilt at being responsible for the caregiver's hardship. ${ }^{3}$

SPB greatly affects the physical and mental health of the patient. In a study by An et al, which mirrored our own findings, it was found that a higher level of CB was associated with higher levels of patient anxiety and depression. Caregivers with higher burden were more likely to report a diminished perceived health status as well as a lower sense of coherence, thus compromising the quality of caregiving. In contrast, patients whose caregivers reported less burden experienced less anxiety and depression symptoms, and a better quality of life, implying that CB plays an important role in influencing a patient's psychological health and quality of life. ${ }^{2}$

Finally, when looking into the relationship between caregiver burden and hospital admissions for the patient, our study found that the odds of hospital admission for the patient increases fourfold when caregivers experience even moderate levels of burden. This is supported by a clinical investigation performed by Ankuda et al, where it was reported that poor caregiver well-being contributed to higher use of healthcare facilities. Caregiving demands, when exceedingly burdensome, are difficult to keep up with, especially in the context of already existing CB. In turn, this leads to worsening of patient health, requiring medical attention and added healthcare expenditures. It was also found that caregiver burnout was linked to an increased reliance on health systems (such as an emergency room) as a source of respite. ${ }^{4}$ The abovementioned findings suggest that identifying caregivers with suboptimal well-being may help to pinpoint patients with impending increases in healthcare costs, which is important given our study's findings that factors related to economic stability were significantly associated with caregiver burden.

\section{Limitations}

A constrained sample size, this being a single-center study, limits the generalizability of results. Other than that, by using pre-validated questionnaires in order to formulate measurable data, the study was limited to responses on an ordinal scale, and did not utilize open-ended questions which may have better elucidated participant experiences. In addition, while including in its pre-requisites for participation an adequate physical condition and mental cognition to answer a questionnaire, the study did not account for possible pre-existing psychological conditions which may influence participant responses. Lastly, being cross-sectional in nature, the study failed to follow participants through a defined time span, painting an incomplete picture of patient and caregiver experiences.

\section{Conclusions}

Educational attainment, employment status and income, all of which influence financial standing, were found to be significantly associated with caregiver burden. Of patient-related factors, older age, a lung primary, and impairments in mobility, self-care and performance of ADLs were identified as associated

Page $14 / 16$ 
factors. Conversely, caregiver burden was found to influence patient quality of life via the impairment of mobility, self-care and ability to perform activities of daily living, as well as an increase in anxiety or depression. Higher levels of caregiver burden were also found to be associated with an increased frequency of hospital admissions for the patient.

With the advancement of cancer therapy prolonging survival, the number of patients requiring continuous care is expected to grow, and caregiver burden will continue to emerge as a significant issue. Early recognition of the physiological and behavioral effects of caring for a family member with cancer provides opportunities to optimize care. Once recognized, strategies for prevention or alleviation may be developed, leaving both patient and caregiver less distressed and better prepared.

\section{RECOMMENDATIONS}

We recommend that future investigation take on a larger scale, increasing the number of participants, and covering other centers providing cancer care, including public hospitals, so as to produce more generalizable results. Likewise, we recommend the selection of equal numbers of participant cancer patients by diagnosis or by chemotherapy regimen, in the hopes of providing a clearer picture of caregiver burden as affected by illness- or treatment-related characteristics. Furthermore, we recommend modifying the study design to prospective, and following participants over time, to evaluate the development of caregiver burden across time. Lastly, we recommend the use of more qualitative data, as in the use of open-ended questions, to allow for more clarity.

This can be performed in cooperation with a psychologist who may further open lines of communication after severe burden is detected.

\section{Declarations}

Funding: This study did not receive any financial support.

Conflicts of interest: The author declares no conflicts of interest.

Availability of data and material: Data was collected via interviews with patients and caregivers at the tertiary institution's Hematology-Oncology Unit.

Code availability: Not applicable

Authors' contributions: This study was written and conducted by the sole author.

Ethics approval: This study was approved by the hospital's Institutional Review Board. The author completed a course on good clinical practice prior to the conduction of the study, presented below.

Consent to participate: Prior to participation via questionnaire completion, the nature of the study was explained by the researcher verbally. These explanations were also presented in writing in the questionnaire form. Consent to disclose information was thereafter sought. 
Consent for publication: The intention to publish the study was also made known to the participants verbally and in writing. The confidentiality of information provided was upheld throughout the conduction of the study.

\section{References}

1. Bevans M, Sternberg EM. Caregiver burden, stress, and health effects among family caregivers of adult cancer patients. Journal of the American Medical Association. 2012 Jan; 307: 398-403.

2. An Y, Fu G, Yuan G. Quality of life in patients with breast cancer: the influence of family caregiver's burden and the mediation of patient's anxiety and depression. The Journal of Nervous and Mental Disease. 2019 Nov; 307: 921-926.

3. Lee JE, Shin DW, Cho J, Yang HK, Kim SY, Yoo HS, Jho HJ, Shin JY, Cho B, Park K, Park JH. Caregiver burden, patient's self-perceived burden, and preference for palliative care among cancer patients and caregivers. Psycho-Oncology. 2015 April; 24: 1545-1551.

4. Ankuda CK, Maust DT, Kabeto MU, McCammon RJ, Langa KM, Levine DA. Association between spousal caregiver well-being and care recipient healthcare expenditures. Journal of the American Geriatrics Society. 2017; 1-7.

5. Dionne-Odom JN, Hull JG, Martin MY, Lyons KD, Prescott AT, Tosteson T, Li Z, Akyar I, Raju D, Bakitas MA. Associations between advanced cancer patients' survival and family caregiver presence and burden. Cancer Medicine. 2016; 5: 853-862.

6. Weitzner MA, Haley WE, Chen $\mathrm{H}$. The family caregiver of the older cancer patient. Hematology/Oncology Clinics of North America. 2000 Feb; 14: 269-281.

7. Ge L, Mordiffi SZ. Factors associated with higher caregiver burden among family caregivers of elderly cancer patients: a systematic review. Cancer Nursing. 2016; 00: 1-8.

\section{Supplementary Files}

This is a list of supplementary files associated with this preprint. Click to download.

- SupplementaryFigure1.pdf

- SupplementaryFigure2.pdf

- SupplementaryTable1.docx

- SupplementaryTable2.docx

- SupplementaryTable3.docx 